\title{
Very low doses of heavy oxygen ion radiation induce premature ovarian failure
}

\author{
Birendra Mishra ${ }^{1,4}$, Ryan Ripperdan ${ }^{1}$, Laura Ortiz ${ }^{1}$ and Ulrike Luderer ${ }^{1,2,3}$ \\ ${ }^{1}$ Department of Medicine, University of California Irvine, Irvine, California, USA, ${ }^{2}$ Department of Developmental \\ and Cell Biology, University of California Irvine, Irvine, California, USA, ${ }^{3}$ Program in Public Health, University of \\ California Irvine, Irvine, California, USA and ${ }^{4}$ Department of Human Nutrition, Food and Animal Sciences, \\ University of Hawaii at Manoa, Honolulu, Hawaii, USA
}

Correspondence should be addressed to U Luderer; Email: uluderer@uci.edu

\begin{abstract}
Astronauts are exposed to charged particles during space travel, and charged particles are also used for cancer radiotherapy. Premature ovarian failure is a well-known side effect of conventional, low linear energy transfer (LET) cancer radiotherapy, but little is known about the effects of high LET charged particles on the ovary. We hypothesized that lower LET (16.5 keV/ $\mathrm{mm})$ oxygen particles would be less damaging to the ovary than we previously found for iron $(\mathrm{LET}=179 \mathrm{keV} / \mu \mathrm{m})$. Adult female mice were irradiated with 0 , 5,30 or 50 cGy oxygen ions or 50 cGy oxygen plus dietary supplementation with the antioxidant alpha lipoic acid (ALA). Six-hour after irradiation, percentages of ovarian follicles immunopositive for $\gamma \mathrm{H} 2 \mathrm{AX}$, a marker of DNA double strand breaks, 4-HNE, a marker of oxidative lipid damage and BBC3 (PUMA), a proapoptotic BCL-2 family protein, were dose dependently increased in irradiated mice compared to controls. One week after irradiation, numbers of primordial, primary and secondary follicles per ovary were dose dependently decreased, with complete absence of follicles in the 50 cGy groups. The $\mathrm{ED}_{50}$ for primordial follicle destruction was $4.6 \mathrm{cGy}$ for oxygen compared to $27.5 \mathrm{cGy}$ for iron in our previous study. Serum FSH and LH concentrations were significantly elevated in 50 cGy groups at 8 week. Supplementation with ALA mitigated the early effects, but not the ultimate depletion of ovarian follicles. In conclusion, oxygen charged particles are even more potent inducers of ovarian follicle depletion than charged iron particles, raising concern for premature ovarian failure in astronauts exposed to both particles during space travel. Reproduction (2017) 154 123-133
\end{abstract}

\section{Introduction}

Temporary amenorrhea and premature ovarian failure are common side effects of therapeutic photon ( $\mathrm{x}$ or $\gamma$-radiation) radiation to the whole body or pelvis (Wallace et al. 1989, Meirow \& Nugent 2001, Lo Presti et al. 2004). Photon radiation damages ovarian follicles at all stages of development, but the quiescent primordial follicles are most sensitive to destruction (Kim \& Lee 2000, Lee et al. 2000, Lee \& Yoon 2005). Because the pool of primordial follicles is believed to be finite, destruction of primordial follicles accelerates the onset of ovarian failure. Premature ovarian failure causes early loss of fertility, as well as increased risk of osteoporosis, cardiovascular disease and Alzheimer's disease (Silva et al. 2001, Dubey et al. 2005, Molina et al. 2005, Shuster et al. 2008, Svejme et al. 2012).

Biological effects of ionizing radiation depend on dose, dose rate and quality of the radiation (ICRP 2003). Linear energy transfer (LET) is an important element of radiation quality. Most prior studies have examined the effects of low LET photon radiation, while very few studies have examined the effects of high LET radiation, such as high charge and energy (HZE) particles, on the ovary. Low LET radiation produces ionization sparsely inside cells, whereas high LET radiation produces dense ionization and is thought to be more destructive (Sridharan et al. 2015, Tokuyama et al. 2015). Both low LET proton ions and high LET carbon ions have been increasingly used in cancer therapy due to the ability to deliver high doses to the tumor while relatively sparing surrounding tissues (Suit et al. 2010). Exposure to charged particle radiation from solar particle events and galactic cosmic rays (GCR) will also be an important occupational hazard for astronauts on deep space missions, such as a mission to Mars. This is of increasing concern as women now make up half of recent National Aeronautics and Space Administration (NASA) astronaut classes (Ronca et al. 2014). GCRs consist of $85 \%$ low LET protons, $14 \%$ helium ions and 1\% HZE particles (Bourdarie \& Xapsos 2008). However, HZE particles, such as oxygen and iron, will make up $21 \%$ of the estimated ionizing dose equivalent from GCR exposure during deep space travel (Slaba et al. 2015). The estimated ionizing dose equivalent of a three-year Mars 
mission is 40 cGy (1.07 Sv absorbed dose) (Cucinotta \& Durante 2006, Barcellus-Hoff et al. 2015).

We recently reported that high LET charged iron particles $(\mathrm{LET}=179 \mathrm{keV} / \mu \mathrm{m})$ induce apoptosis, oxidative damage and DNA double strand breaks in oocytes and granulosa cells of ovarian follicles, depleting nearly all of the primordial follicle pool at doses of 30 and 50cGy (Mishra et al. 2016). We also showed that dietary supplementation with the antioxidant alpha lipoic acid (ALA) was partially protective against the early ovarian effects of iron ions, but was not able to prevent premature ovarian failure. We hypothesized that oxygen charged particles, with LET of $16.5 \mathrm{keV} /$ $\mu \mathrm{m}$, would be less potent inducers of ovarian follicle destruction than iron charged particles. We established the dose-response relationships for induction of ovarian follicle DNA double strand breaks, oxidative damage and apoptosis and follicle depletion by charged oxygen particles and tested ALA supplementation as a potential protective countermeasure.

\section{Materials and methods}

\section{Animals}

Twelve-week-old female mice (C57BL/6) from Jackson Laboratories, $\quad N=8$ /experimental group) were randomly assigned to experimental group. Mice were irradiated with $0,5,30$ or 50 cGy charged oxygen particles (LET $=16.5 \mathrm{keV} /$ $\mu \mathrm{m})$ at energy of $600 \mathrm{MeV} / \mu$ and dose rates of $12.5-44.3 \mathrm{cGy} /$ min. Two groups were irradiated at the highest dose, one fed AIN-93M diet and the other fed the same diet supplemented with $150 \mathrm{mg} / \mathrm{kg}$ diet of the antioxidant ALA (ALA from Sigma Aldrich; diets formulated by Bio-Serv, Flemington, NJ, USA), beginning one week before irradiation and continuing until euthanasia. Irradiations were performed at the NASA Space Radiation Laboratory, Brookhaven National Laboratory, NY, USA (La Tessa et al. 2016). Mice for the 0cGy group were transported and restrained identically to the irradiated groups. All animal procedures were approved by the Institutional Animal Care and Use Committees at Brookhaven National Laboratory and the University of California Irvine. Mice were killed by $\mathrm{CO}_{2}$ inhalation at $6 \mathrm{~h}, 1$ week and 8 weeks after irradiation. Ovaries and blood were collected at the time of euthanasia.

\section{Vaginal cytology}

Estrous cycling was monitored every morning by microscopic examination of fresh vaginal lavage fluid obtained in $0.9 \%$ sodium chloride (Cooper et al. 1993) beginning at 6 weeks after irradiation and continuing for at least 3 estrous cycles or 14 days if the mice were not cycling. Mice were housed individually for one week before and while monitoring estrous cycles and were killed on the day of metestrus of the estrous cycle at approximately 8 weeks after irradiation. Estrous cycles were not monitored in the mice killed at the acute, $6 \mathrm{~h}$ and 1 week time points because we wished to kill the mice as close to the designated acute time point as possible.

\section{Ovarian histomorphometric analysis}

Ovaries were fixed in Bouin's fixative (Fisher Scientific), washed in $50 \%$ ethanol, stored in $70 \%$ ethanol, embedded in paraffin and serially sectioned at $5 \mu \mathrm{m}$ thickness. Sections were stained with Gill's hematoxylin (Fisher Scientific) and eosin Y (Sigma Aldrich). Ovarian follicles were counted blind to treatment group using an Olympus BX60 light microscope equipped with

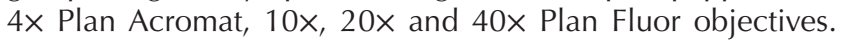
Images were captured using a Retiga 2000R cooled CCD digital camera system and QCapture Pro software (QImaging). Ovarian follicles were classified as primordial (single layer of flattened granulosa cells), primary (single layer of cuboidal or mixed cuboidal/flattened granulosa cells), secondary (more than one layer of granulosa cells) or antral (multiple layers of granulosa cells and possessing an antral space or spaces) and were further classified as healthy or atretic as described previously (Lopez \& Luderer 2004, Lim et al. 2013). Atretic secondary and antral follicles were identified by the presence of 3 or more pyknotic granulosa cells per largest cross section and separation of the oocyte from the granulosa cells. Atretic primordial and primary follicles were identified by shrunken, eosinophilic oocytes. Primordial, primary and secondary follicles were counted in every fifth serial section. The counts were multiplied 5 times to obtain estimates of the total number of follicles per ovary. To avoid over counting, primordial and small primary follicles were only counted if the oocyte nucleus was clearly visible; larger primary and secondary follicles were only counted if the oocyte nucleolus was clearly visible. Antral follicles were followed through every section taking care to count each antral follicle only once.

\section{Immunohistochemistry}

Ovaries were fixed in $4 \%$ paraformaldehyde (Electron Microscopy Sciences) in PBS, cryoprotected in 15\% sucrose (Fisher Scientific), embedded in optimal cutting temperature (OCT) embedding compound (Electron Microscopy Sciences) and stored at $-80^{\circ} \mathrm{C}$ until sectioning. Ovaries were serially sectioned at $7 \mu \mathrm{m}$ thickness and slides were stored at $-80^{\circ} \mathrm{C}$ until staining. Slides were immunostained using primary antibodies to phosphorylated $\mathrm{H} 2 \mathrm{~A}$ histone family, member $\mathrm{X}(\gamma \mathrm{H} 2 \mathrm{AX})$, 4-hydroxynonenal (4-HNE), BCL2 binding component 3 (BBC3), also called p53 upregulated modulator of apoptosis or PUMA and secondary antibody as detailed in Table 1. Diaminobenzidine substrate (Roche) was used for visualization of antibody binding, and sections were counterstained with Gill's hematoxylin. Scoring was done blind to treatment group as described previously (Mishra et al. 2016). The percentages of follicles with positive granulosa cells or positive oocytes per section were calculated, and the percentages from the six scored sections per ovary were averaged for analysis.

\section{Measurement of hormones}

Serum concentrations of follicle-stimulating hormone (FSH) and luteinizing hormone (LH) were measured 8 weeks after irradiation using Millipore Pituitary Panel Multiplex kits at the Center for Research in Reproduction, University of Virginia, Charlottesville, VA, USA. The intra- and inter-assay coefficients of variation were $5.5 \%$ and $11.5 \%$ respectively.

www.reproduction-online.org 
Table 1 Antibodies used for immunohistochemistry.

\begin{tabular}{|c|c|c|c|c|c|}
\hline Protein target & Name of antibody & Manufacturer/catalog & $\begin{array}{l}\text { Species raised in; } \\
\text { monoclonal or polyclonal }\end{array}$ & Dilution & $\begin{array}{c}\text { Secondary antibody } \\
\text { dilution }\end{array}$ \\
\hline Phospho-histone H2A.X & Anti- $\gamma \mathrm{H} 2 \mathrm{AX}$ & Cell Signaling, $\# 9718$ & Rabbit; monoclonal & $1: 200$ & $1: 200$ \\
\hline $\begin{array}{l}\text { p53 upregulated modulator } \\
\text { of apoptosis }\end{array}$ & Anti-PUMA & Abcam, \#ab9643 & Rabbit polyclonal & $1: 200$ & $1: 400$ \\
\hline 4-Hydroxynonenal adducts & Anti-4-hydroxy-2-nonenal & $\alpha$ Diagnostics, \#HNE11-S & Rabbit; Polyclonal & $1: 700$ & $1: 200$ \\
\hline
\end{tabular}

\section{Statistical analyses}

All data are presented as the mean \pm S.E.M. in figures. Differences among treatment groups were analyzed by analysis of variance (ANOVA) followed by post hoc LSD test (for equal variances) or Dunnett T3 test (unequal variances) for follicle counts or hormone concentrations. Levene's test was used to check for equality of variances. Differences among groups for endpoints expressed as percentages were analyzed by nonparametric Kruskal-Wallis test followed by Mann-Whitney $U$ test for intergroup comparisons. Statistical analyses were carried out using SPSS 20 (Windows) or SPSS 23 (Macintosh; IBM Corporation).

\section{Results}

\section{Charged oxygen particles increase ovarian histone 2AFX phosphorylation}

We assessed DNA double strand breaks using $\gamma \mathrm{H} 2 \mathrm{AX}$ immunostaining $6 \mathrm{~h}$ after irradiation with charged oxygen particles. At $6 \mathrm{~h}$, the percentages of primary, secondary and antral follicles with $\gamma \mathrm{H} 2 \mathrm{AX}$-positive granulosa cells increased in a dose-dependent manner $(P<0.005$, effect of group by Kruskal-Wallis test; Fig. 1A, B, C and E). The percentages of primordial, primary and secondary follicles with $\gamma \mathrm{H} 2 \mathrm{AX}$-positive oocytes also increased in a dose-dependent manner $(P<0.007$, effect of group; Fig. 1A, B, C and F; Fig. 2 top). ALA supplementation did not mitigate these increases in $\gamma \mathrm{H} 2 \mathrm{AX}$ immunostaining after 50 cGy oxygen irradiation.

\section{Charged oxygen particles increase ovarian oxidative stress}

We assessed oxidative lipid damage $6 \mathrm{~h}$ after irradiation with charged oxygen particles using immunostaining for 4-HNE (Mishra et al. 2016). At $6 \mathrm{~h}$ after irradiation with charged oxygen particles, the percentages of primary, secondary and antral follicles with 4-HNE-positive granulosa cells were dose dependently increased $(P<0.03$, effect of group by Kruskal Wallis test, Fig. 3A, $\mathrm{B}, \mathrm{C}$ and $\mathrm{E})$. At $6 \mathrm{~h}$, the percentages of primordial, primary, secondary and antral follicles with 4-HNEpositive oocytes also increased in a dose-dependent manner $(P<0.04$, Fig. 3A, B, C and F; Fig. 2 middle). ALA supplementation partially mitigated the effects of 50cGy oxygen irradiation on lipid peroxidation in granulosa cells of secondary follicles and oocytes of primordial follicles (Fig. 3E and F).

\section{Charged oxygen particles increase ovarian follicular apoptosis}

Because acute increases in PUMA (proapoptotic BCL-2 family member) are required for $\gamma$-radiation-induced germ cell death in neonatal ovaries (Kerr et al. 2012), and we observed that PUMA increased in iron ionirradiated adult ovaries together with caspase 3 activation (Mishra et al. 2016), we assessed follicle apoptosis at $6 \mathrm{~h}$ post irradiation using PUMA immunostaining. The percentages of secondary and antral follicles with PUMA-positive granulosa cells were dose dependently elevated at $6 \mathrm{~h}$ after oxygen irradiation $(P<0.02$, effect of group by Kruskal-Wallis test; Fig. 4A, B, C and E). The percentages of primordial, primary, secondary and antral follicles with PUMA-positive oocytes were also dose dependently increased at $6 \mathrm{~h} \quad(P<0.005$; Fig. 4A, B, C and F; Fig. 2 bottom). The increases in PUMA levels after charged oxygen irradiation in secondary follicle oocytes and granulosa cells and antral follicle granulosa cells were mitigated by ALA supplementation (Fig. 4E and F).

\section{Charged oxygen particles disrupt estrous cycling}

Estrous cycling was monitored by vaginal cytology from 6 to 8 weeks after irradiation. $100 \%$ of mice in both 50 cGy exposure groups, $75 \%$ of mice in the 30 cGy group and $37.5 \%$ of mice in the 5 and 0 cGy groups had irregular estrous cycles, defined as not having regular cycles of 4-5 day length $(P=0.003$, differences among groups by Fisher's exact test). $37 \%$ of the mice in the 50 cGy groups did not cycle at all, while all the mice in the 0,5 and 30cGy groups cycled, whether regularly or irregularly (Table 2). For the mice that were cycling, cycle lengths tended to be longer in the mice in the 30 and 50 cGy groups compared to the 0 and 5 cGy groups $(P<0.001$, effect of group; Table 2), with the 30cGy group having significantly longer cycles than the 0cGy group. However, there were no statistically significant differences among groups in the percentages of days with predominantly cornified cytology, characteristic of estrus or with predominantly leukocytic cytology, characteristic of metestrus and diestrus (Table 2).

\section{Charged oxygen particles deplete ovarian follicles}

To examine the consequences of DNA damage, oxidative lipid damage and apoptosis induction by 
A
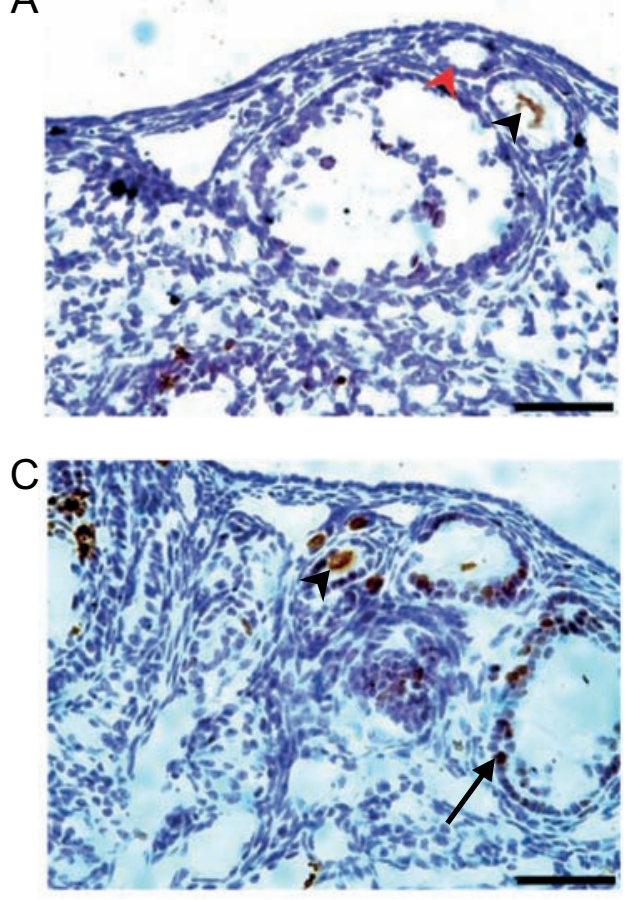

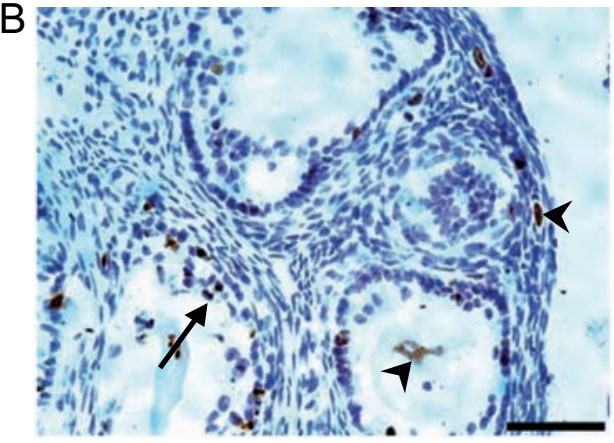

D

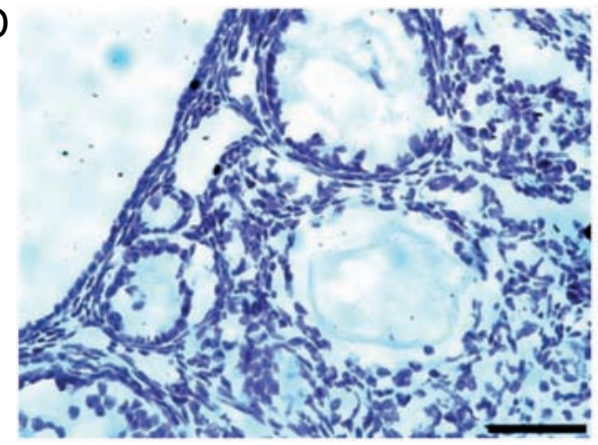

$\mathrm{E}$

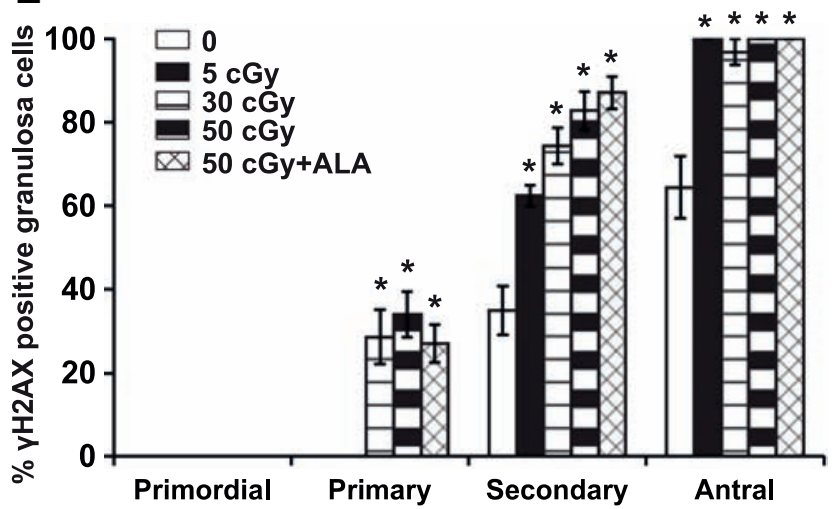

$\mathrm{F}$

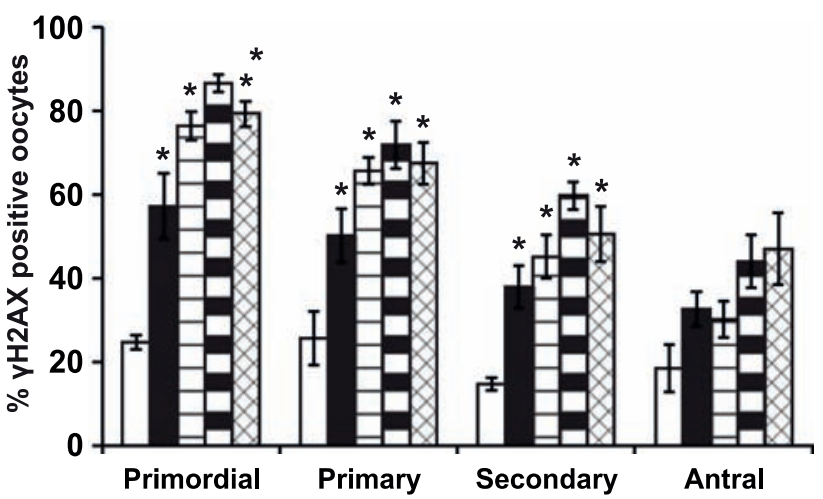

Figure 1 Charged oxygen particles increase $\gamma \mathrm{H} 2 \mathrm{AX}$ immunostaining in ovarian follicles. Mice in this and subsequent figures were fed AIN-93M diet or diet supplemented with $150 \mathrm{mg} / \mathrm{kg}$ ALA from one week before irradiation with the indicated doses of charged oxygen particles until euthanasia. Double strand DNA damage was analyzed using $\gamma \mathrm{H} 2 \mathrm{AX}$ immunostaining in ovarian sections $6 \mathrm{~h}$ after irradiation. Representative images of $\gamma \mathrm{H} 2 \mathrm{AX}$ immunostaining in 0 (A), 5 (B), 50 cGy (C) ovaries and lack of immunostaining in negative control with primary antibody replaced by nonimmune IgG (D). Cells are indicated by black (positively stained) or red (negatively stained) arrows (granulosa) and arrowheads (oocytes). Scale bars, $50 \mu \mathrm{m}$. Graphs show the mean \pm S.E.M. percentage of follicles with $\gamma \mathrm{H} 2 \mathrm{AX}$-positive granulosa cells or oocytes. (E) There were statistically significant differences in percentages of primary, secondary and antral follicles with $\gamma \mathrm{H} 2 \mathrm{AX}$-positive granulosa cells among groups $(P<0.005$, Kruskal Wallis tests). (F) There were statistically significant differences in percentages of primordial, primary, and secondary follicles with $\gamma \mathrm{H} 2 \mathrm{AX}$-positive oocytes $\left(P<0.007\right.$, Kruskal-Wallis tests). ${ }^{*} P<0.05$ vs 0 cGy control by Mann-Whitney test. $N=4$ mice/group.

oxygen-charged particles in ovarian follicles, we counted healthy primordial, primary, secondary and antral follicles at 1 week after irradiation. The numbers of healthy primordial $(P<0.001$, effect of group by Kruskal-Wallis test; Fig. 5A), primary $(P<0.001$; Fig. 5B) and secondary $(P=0.002$, Fig. $5 \mathrm{C})$ follicles per ovary were dose dependently decreased in mice irradiated with oxygen. The numbers of healthy antral follicles were not affected by charged oxygen irradiation, but we had limited power to detect the differences because of the large inter-mouse variability in this endpoint. ALA supplementation did not mitigate the depletion of ovarian follicles 1 week after irradiation with 50cGy charged oxygen particles. 

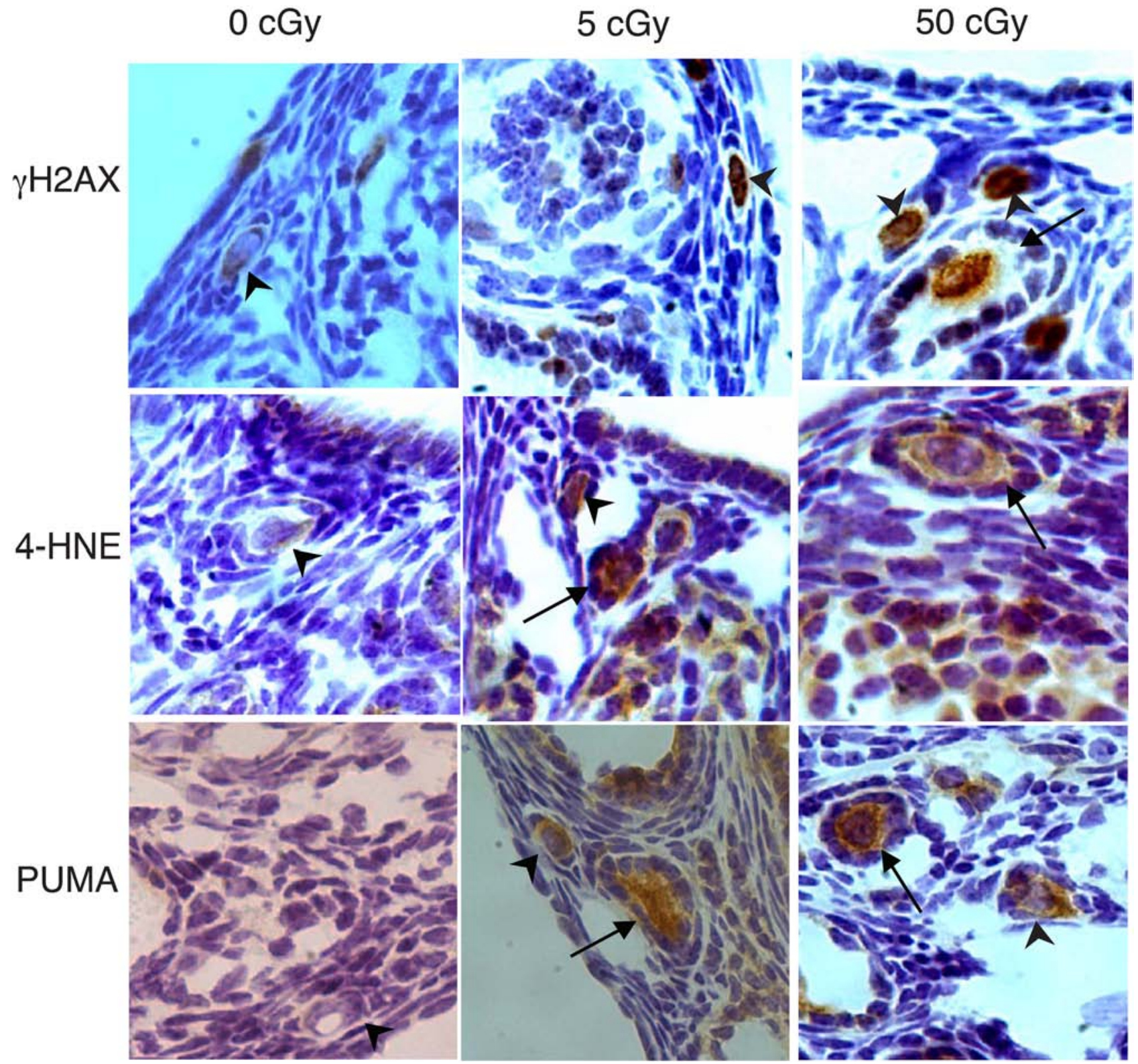

Figure 2 Representative $\gamma \mathrm{H} 2 \mathrm{AX}$, 4-HNE and PUMA immunostaining in primordial and primary follicles after irradiation with charged oxygen particles. Processing of ovaries and immunostaining were performed as described in methods. Representative images of ovarian sections immunostained with antibodies to $\gamma \mathrm{H} 2 \mathrm{AX}$ (top row), 4-HNE (middle row) or PUMA (bottom row). Doses of charged oxygen particles are indicated at top. Arrowheads indicate primordial follicles and arrows indicate primary follicles Original magnification $400 \times$.

\section{Charged oxygen particles are more potent destroyers of primordial follicles than charged iron particles}

We analyzed the dose-response for primordial follicle depletion by oxygen ions (data from the present study) and iron ions (data from Mishra et al. 2016) using linear regression on the log transformed follicle counts. We then calculated the $\mathrm{ED}_{50}$ for primordial follicle depletion at 1 week after irradiation. The $\mathrm{ED}_{50}$ was $27.5 \mathrm{cGy}$ for iron and 4.6cGy for oxygen, indicating that charged oxygen particles with LET $16.5 \mathrm{keV} / \mu \mathrm{m}$ are more potent destroyers of primordial follicles than charged iron particles with LET of $179 \mathrm{keV} / \mu \mathrm{m}$.

\section{Charged oxygen particles alter reproductive hormone concentrations}

If the hypothalamic-pituitary-ovarian axis were functioning normally, one would expect the depletion of ovarian follicles to result in decreased negative feedback to the hypothalamus and pituitary, increasing 

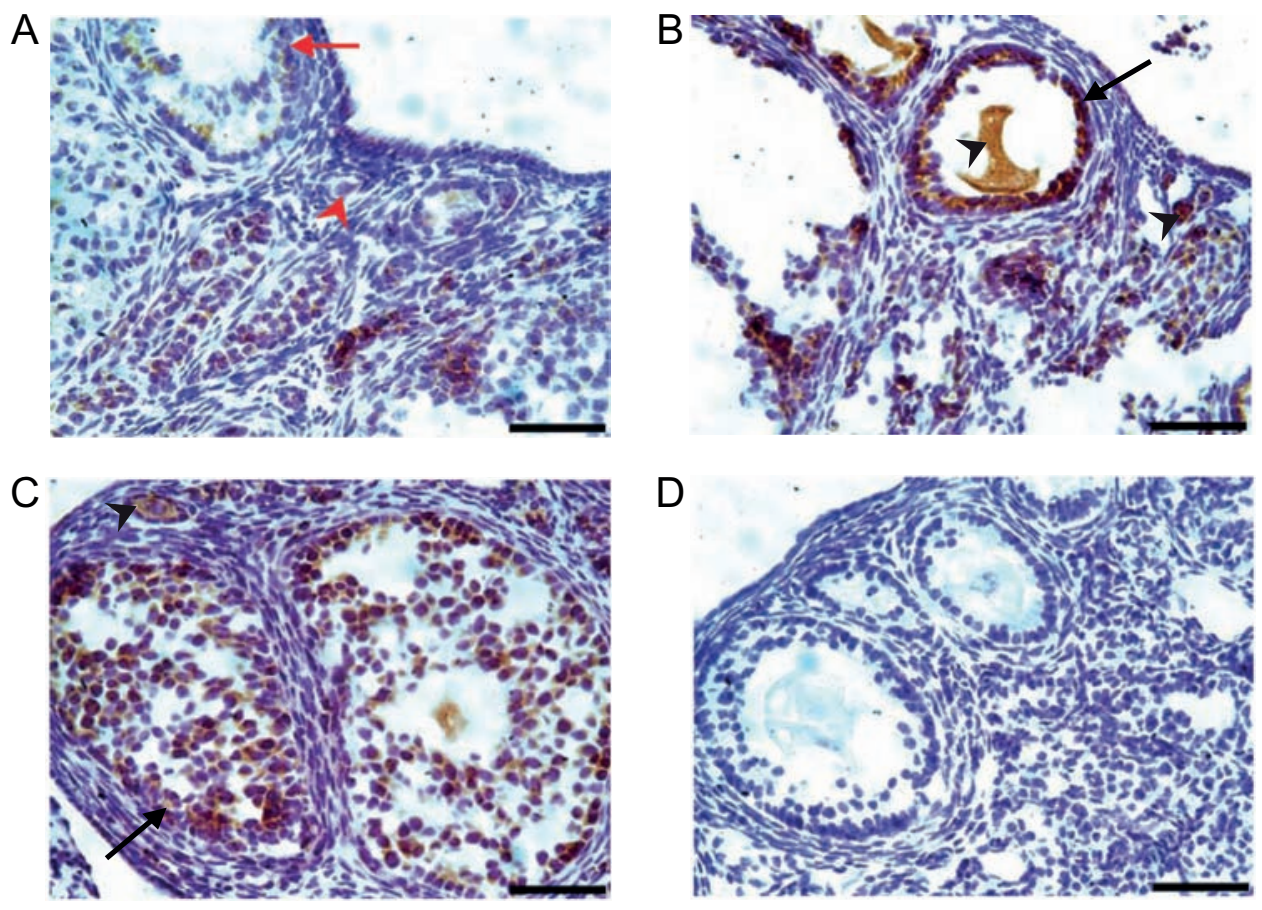

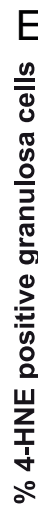
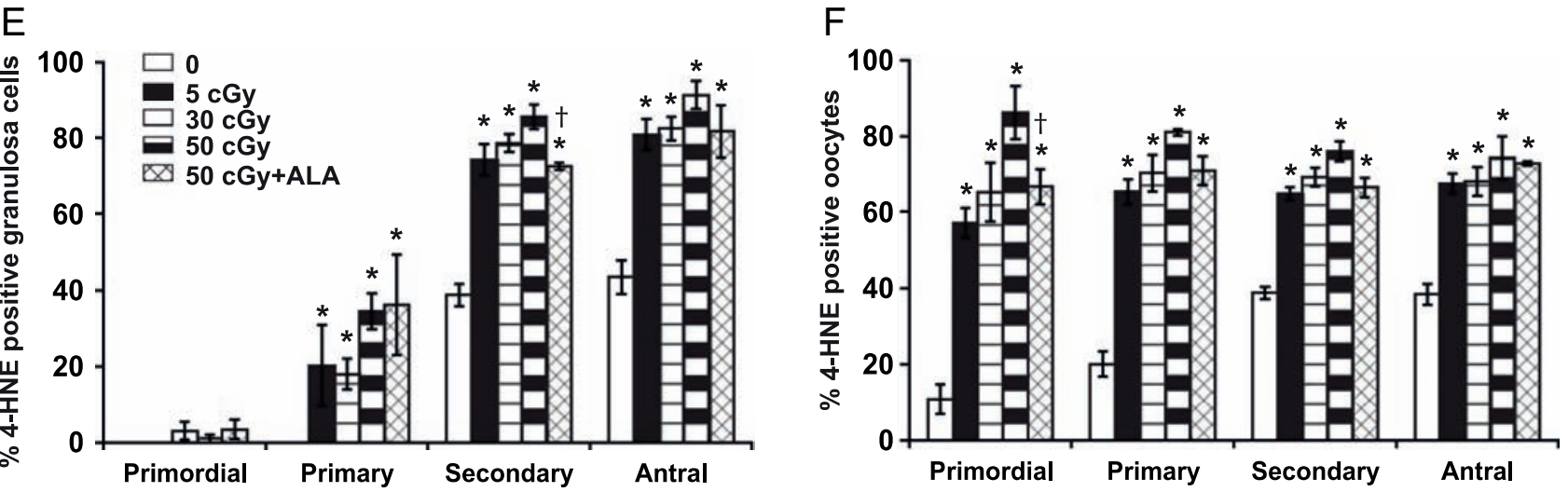

Figure 3 Charged oxygen particles increase lipid peroxidation in ovarian follicles. Lipid peroxidation was analyzed using 4-HNE immunostaining in ovarian sections $6 \mathrm{~h}$ after irradiation. Representative images of 4-HNE immunostaining in 0 (A), 5 (B), $50 \mathrm{cGy}(\mathrm{C})$ ovaries and lack of immunostaining in negative control ovary with primary antibody replaced by nonimmune IgG (D). Representative positively stained cells are indicated by black arrows (granulosa) and arrowheads (oocytes) and negatively stained by red arrows/arrowheads. Scale bars, $50 \mu \mathrm{m}$. Graphs show the means \pm S.E.M. of percentages of follicles with 4-HNE positive granulosa cells or oocytes. (E) Percentages of follicles with 4-HNE positive granulosa cells varied significantly among groups for primary, secondary, and antral follicles $(P<0.03$, Kruskall-Wallis test). (F) Percentages of follicles with 4-HNE positive oocytes varied significantly among groups for primordial, primary, secondary, and antral follicles $\left(P<0.04\right.$, Kruskall-Wallis test). ${ }^{*} P<0.05$ vs 0 cGy control by Mann-Whitney test. ${ }^{\dagger} P<0.05$ vs 50 cGy. $N=4$ mice/group.

the circulating levels of follicle-stimulating hormone $(\mathrm{FSH})$ and luteinizing hormone (LH) secreted from the anterior pituitary gland. Consistent with this, serum $\mathrm{FSH}$ and $\mathrm{LH}$ concentrations increased with charged oxygen particle dose 8 weeks after irradiation $(P<0.001$, effects of treatment group; Fig. $6 \mathrm{~A}$ and $B)$. Mice irradiated with $50 \mathrm{cGy}$ oxygen fed ALA supplemented diet had similar levels of FSH and LH as the 50 cGy iron group fed nonsupplemented diet (Fig. 6A and B).

\section{Discussion}

High LET radiation causes dense ionization, which is believed to be more damaging to DNA than low LET radiation. Women are exposed to high LET charged particle radiation during travel in deep space and ion beam radiotherapy; however, the ovarian effects of most charged particles have not been studied. Our results reveal that acute exposure to charged oxygen particles dose dependently increased DNA damage 

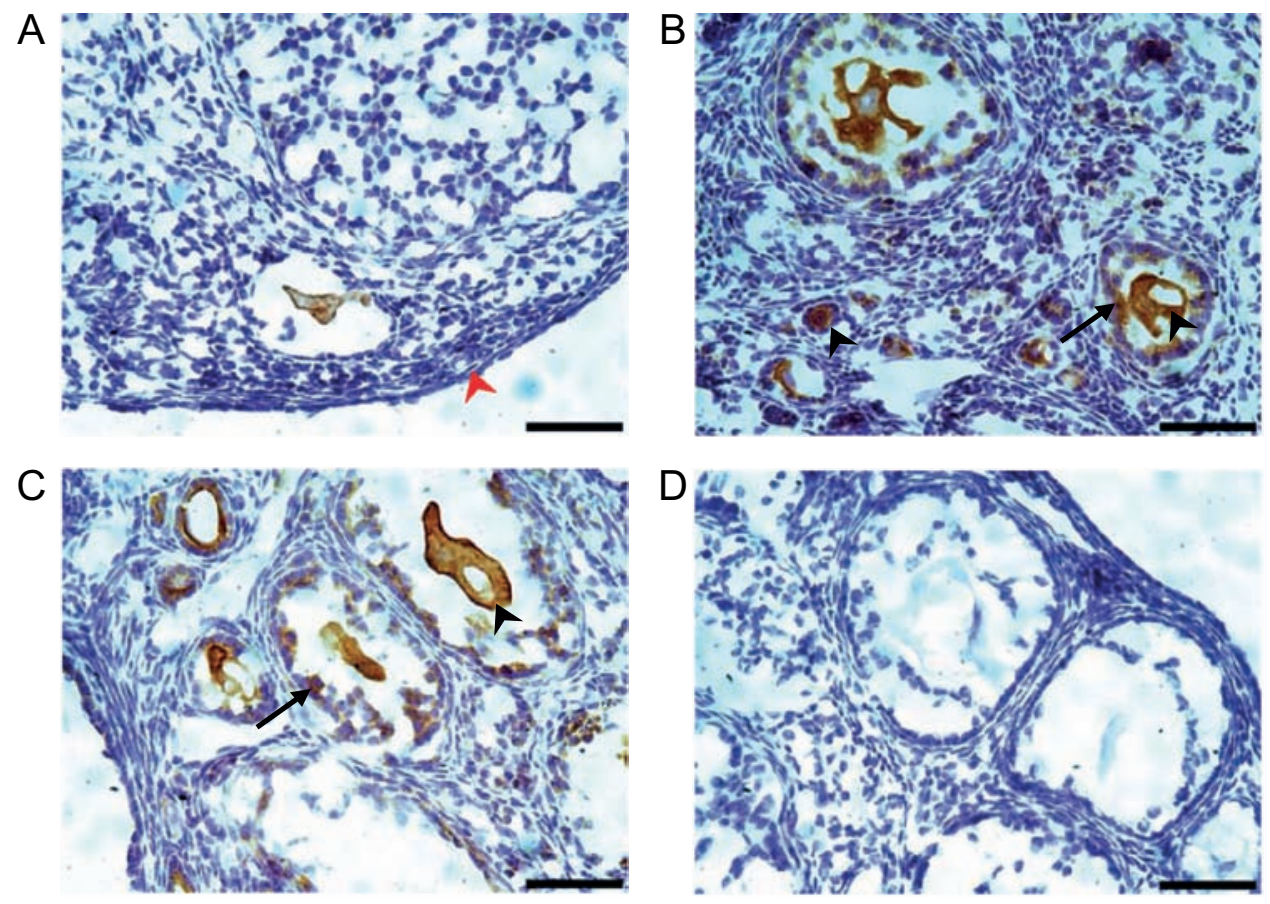

E

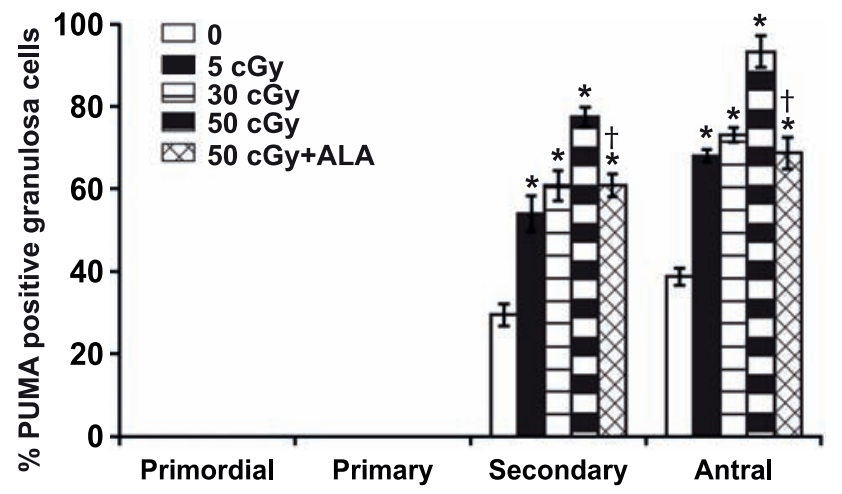

\section{$\mathrm{F}$}

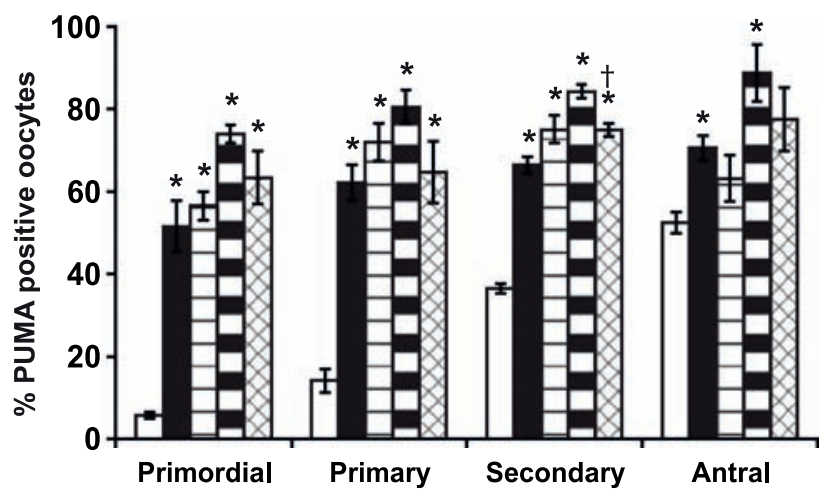

Figure 4 Induction of the proapoptotic protein PUMA in ovarian follicles by charged oxygen particles. Representative images of PUMA immunostaining in 0 (A), 5 (B), 50 cGy (C) ovaries and lack of immunostaining in negative control ovary with primary antibody replaced by nonimmune $\operatorname{lgG}(\mathrm{D})$. Representative positively stained cells are indicated by black arrows (granulosa) and arrowheads (oocytes), while negatively stained are indicated by red arrows/arrowheads. Scale bars, $50 \mu \mathrm{m}$. Graphs show the means \pm S.E.M. of percentages of follicles with PUMA-positive granulosa cells or oocytes at $6 \mathrm{~h}$ after irradiation. (E) Percentages of secondary and antral follicles with PUMA-positive granulosa cells varied significantly among groups $(P<0.05$, Kruskal-Wallis test). (F) Percentages of primordial, primary, secondary, and antral follicles with PUMA-positive oocytes varied significantly among groups $\left(P<0.05\right.$, Kruskal-Wallis). ${ }^{*} P<0.05$ vs 0 cGy control by Mann-Whitney test. ${ }^{\dagger} P<0.05$ vs 50 cGy. $N=4$ mice/group.

Table 2 Effects of charged oxygen particles on estrous cycle length.

\begin{tabular}{lcccc}
\hline Group (cGy) & Number $(\%)$ cycling & Cycle length \pm s.E.M. (days)* & Cornified cytology (\% of days) & Leukocytic cytology (\% of days) \\
\hline 0 & $8(100)$ & $4.0 \pm 0.1$ & $41.9 \pm 2.5$ & $37.9 \pm 2.5$ \\
5 & $8(100)$ & $4.3 \pm 0.2$ & $35.9 \pm 2.3$ & $41.6 \pm 2.8$ \\
30 & $8(100)$ & $5.2 \pm 0.3 * *$ & $40.8 \pm 6.7$ & $38.5 \pm 3.5$ \\
50 & $5(63)$ & $6.1 \pm 0.6$ & $31.7 \pm 4.9$ & $48.6 \pm 2.5$ \\
50 plus ALA & $5(63)$ & $5.5 \pm 0.5$ & $31.5 \pm 7.1$ & $42.8 \pm 6.7$ \\
\hline
\end{tabular}

*Includes only mice that were cycling; ${ }^{* *} P<0.05$ vs 0 cGy by Dunnett T3 test. 

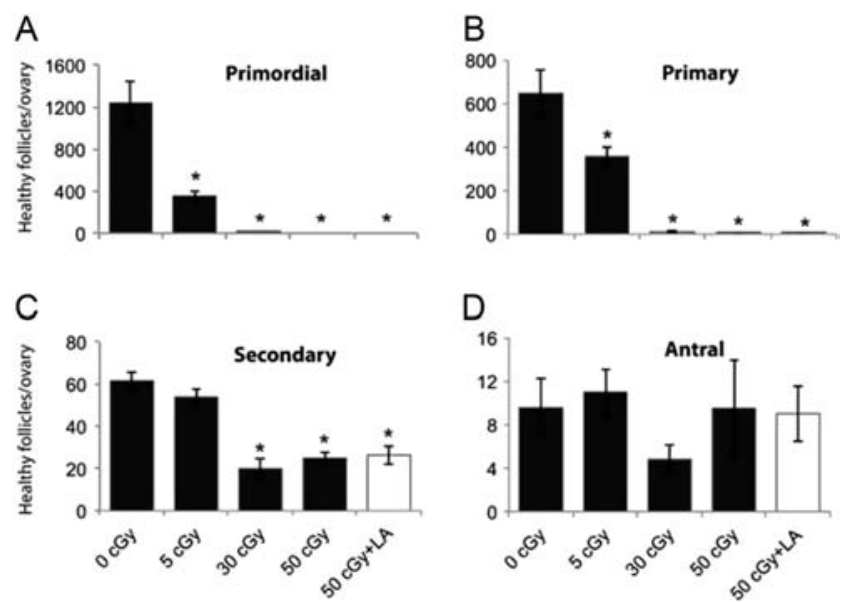

Figure 5 Charged oxygen particles deplete ovarian follicles. Ovaries were processed for histology, and follicles were classified and counted as described in 'Methods' section ( $N=4-5$ mice/group). Graphs show the mean \pm S.E.M. of number healthy follicles of the indicated stages of development per ovary. (A) Primordial follicles, $P<0.001$, effect of group by Kruskal Wallis test. (B) Primary follicles, $P<0.001$, effect of group by Kruskal Wallis test. (C) Secondary follicles, $P=0.002$, effect of group by Kruskal-Wallis test. (D) Antral follicles, effect of group not statistically significant. ${ }^{*} P<0.05$, compared to 0 cGy control by Mann-Whitney test.
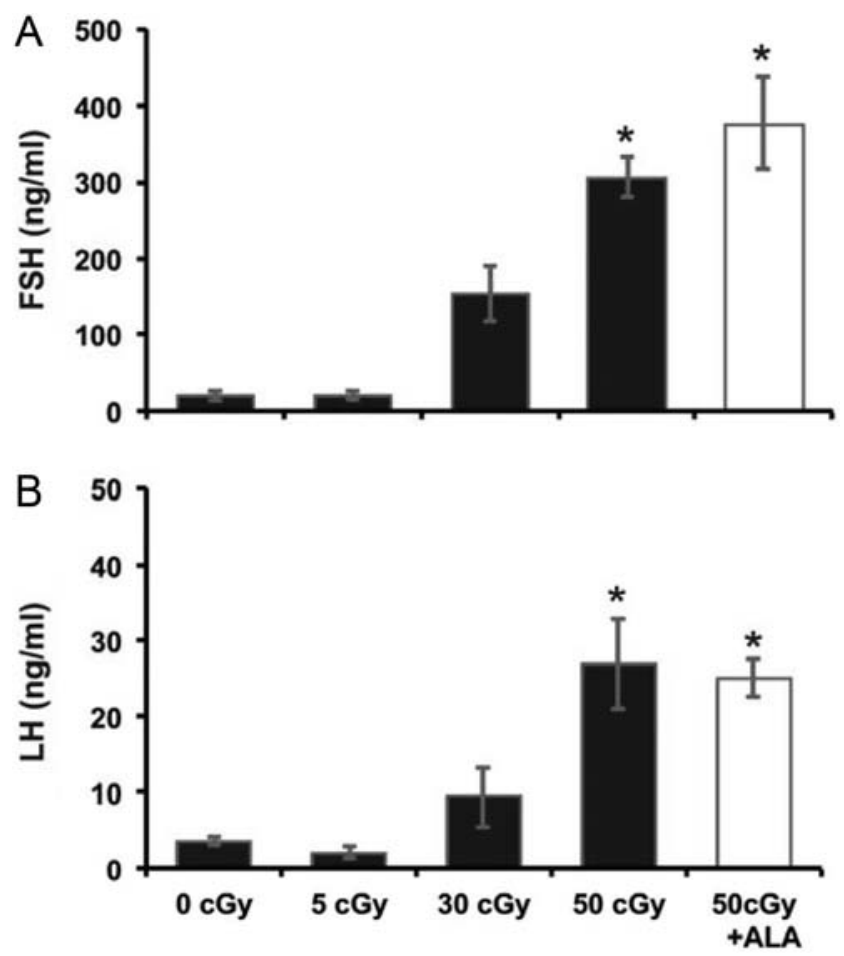

Figure 6 Charged oxygen particles decrease ovarian negative feedback to the hypothalamus and pituitary. Graphs show the mean \pm S.E.M. of serum FSH (A) or LH (B) levels at 8 week after irradiation ( $N=6-8$ mice/group). There were significant differences in FSH and $\mathrm{LH}$ concentrations among treatment groups $(P<0.001)$. $* P<0.05$, compared to 0 cGy control. $(\gamma \mathrm{H} 2 \mathrm{AX}$ immunostaining), oxidative lipid damage (4-HNE immunostaining) and apoptosis (PUMA immunostaining) in ovarian follicles at $6 \mathrm{~h}$, resulting in depletion of ovarian follicles at 1 week, irregular estrous cycling and elevated serum concentrations of $\mathrm{LH}$ and FSH due to loss of ovarian negative feedback. Ovarian follicle numbers were dose dependently depleted at 1 week post irradiation, with complete absence of primordial and near-complete absence of primary follicles after irradiation with 30 cGy and 50 cGy oxygen ions. Dietary supplementation with the antioxidant ALA was not protective against ovarian follicle depletion caused by exposure to 50 cGy charged oxygen particles. Our results also show that charged oxygen particles are more potent destroyers of primordial follicles than charged iron particles (Mishra et al. 2016).

Depletion of ovarian follicles and resultant premature ovarian failure is a significant adverse side effect of $\gamma$-irradiation for cancer treatment (Wallace et al. 1989, Lee et al. 2000, Wallace et al. 2003, Lee \& Yoon 2005, Lo Presti et al. 2004). The $\mathrm{ED}_{50}$ for follicle depletion in humans by $\gamma$-radiation has been estimated to be less than 2 Gy (Wallace et al. 2003). The $\mathrm{ED}_{50}$ for follicle destruction in 6-week-old ICR mice 1 week after irradiation is less than 60 cGy $(10 \%$ of total follicles remaining) (Mathur et al. 1991) and in 6-week-old C57BL6/DBA F1 hybrid mice 24h after irradiation is less than 50 cGy (40\% of primordial follicles remaining) (Pesty et al. 2009). These doses were the lowest tested in both studies, and the data do not permit accurate estimation of $\mathrm{ED}_{50}$. Using data from the current study and from our prior study (Mishra et al. 2016), we estimated the $\mathrm{ED}_{50}$ for primordial follicle depletion at one week after irradiation as $27.5 \mathrm{cGy}$ for charged iron particles and 4.6cGy for charged oxygen particles. These results suggest that oxygen and perhaps iron ion radiation have greater relative biological effectiveness for ovarian follicle destruction than $\gamma$-radiation, but more detailed dose-response data comparing $\gamma$-radiation and charged particle radiation side by side in mice of the same strain and age are necessary to confirm this. The greater potency of lower LET oxygen compared to higher LET iron was unexpected, but can perhaps be explained by the relationship between LET and fluence (number of particles received by a surface per unit area). At LET of $16.5 \mathrm{keV} / \mu \mathrm{m}$, oxygen has a fluence of about $10^{-7}$ particles $/ \mathrm{cm}^{2}$ second steradian MeV/nucleon, while iron at LET of $179 \mathrm{keV} / \mu \mathrm{m}$ iron has a fluence of $3 \times 10^{-8}$ particles $/ \mathrm{cm}^{2}$ second steradian $\mathrm{MeV} /$ nucleon (ICRP 2003, Bourdarie \& Xapsos 2008). Therefore, at the same radiation dose, more oxygen particles are expected to traverse a primordial follicle than iron particles, causing greater damage.

The molecular mechanisms underlying follicle destruction by radiation exposure are not completely understood. Studies in diverse cell types support that about one-third of the detrimental effects of ionizing 
radiation at the cellular level are due to direct DNA damage and two-thirds are due to generation of reactive oxygen species (ROS) from ionization of water (Spitz et al. 2004, Dayal et al. 2008). Increased ROS persist for hours, days and even weeks post irradiation, reflecting perturbations to the redox homeostasis of cells (Spitz et al. 2004, Dayal et al. 2008). We previously showed that rapid and sustained increases in ROS occurred in human COV434 granulosa cells within $30 \mathrm{~min}$ after 1 or 5 Gy $\gamma$-irradiation, followed by apoptotic death at $6 \mathrm{~h}$, whereas overexpression of glutamate cysteine ligase subunit genes to enhance glutathione synthesis prevented the radiation-induced rise in ROS and prevented apoptotic death of the cells (Cortés-Wanstreet et al. 2009). Consistent with involvement of ROS in follicle destruction, we observed significantly increased immunostaining for 4-HNE, a marker of oxidative lipid damage, in oocytes and/or granulosa cells of follicles at all stages of development $6 \mathrm{~h}$ after exposure to even the lowest dose of charged oxygen particles. Moreover, oxidative DNA damage is a known cause of double strand DNA breaks (Cadet et al. 2012), and we observed increased immunostaining for $\gamma \mathrm{H} 2 \mathrm{AX}$ in oocytes and granulosa cells at $6 \mathrm{~h}$ after oxygen ion irradiation. In our prior work, we observed similar increases in $4-\mathrm{HNE}$ and $\gamma \mathrm{H} 2 \mathrm{AX}$, as well as nitrotyrosine, a marker of oxidative protein damage, in ovarian follicles after irradiation with 50cGy charged iron particles (Mishra et al. 2016). The thiol ALA acts as a direct antioxidant in vitro at concentrations not achievable in vivo (Petersen Shay et al. 2008, Rochette et al. 2013). The in vivo antioxidant activity of ALA is mediated largely by increased cellular uptake of ascorbate and cysteine and by increased translation of the transcription factor NRF2, a master regulator of the antioxidant response (Suh et al. 2004, Petersen Shay et al. 2012, Rochette et al. 2013). The lack of a prominent protective effect of dietary supplementation with ALA against the ovarian effects of 50 cGy oxygen ions is most likely due to overwhelming ROS generation by this relatively high dose that could not be overcome with supplementation of a single antioxidant. Supporting this notion, we observed minor, but statistically significant protective effects of ALA against oxidative lipid damage in secondary and primordial follicles.

The proapoptotic $\mathrm{BH} 3$-only $\mathrm{BCL}-2$ family protein PUMA is required for germ cell apoptosis induction by $\gamma$-radiation in neonatal ovaries (Kerr et al. 2012). We previously showed that charged iron particles increase PUMA protein expression in oocytes and granulosa cells of follicles at $6 \mathrm{~h}$ after irradiation, while caspase 3 activation is only observed in granulosa cells of secondary follicles at that time point (Mishra et al. 2016). Therefore, in this study, we analyzed the expression of PUMA as a marker of follicular apoptosis induction. In the present study, we observed significant dose-dependent increases in PUMA immunostaining in oocytes of all follicle stages and in granulosa cells of secondary and antral follicles $6 \mathrm{~h}$ after oxygen ion irradiation. Moreover, ALA supplementation decreased the magnitude of the effects of 50cGy oxygen irradiation on PUMA expression to the levels observed with 30cGy irradiation. In contrast, ALA supplementation decreased oocyte and granulosa cell PUMA expression in 50 cGy iron ion irradiated mice to levels observed in 0 cGy sham-irradiated mice (Mishra et al. 2016).

Consistent with depletion of ovarian follicles resulting in loss of ovarian negative feedback to the hypothalamus and pituitary, we observed significantly increased serum $\mathrm{LH}$ and FSH concentrations in the 50cGy irradiated groups 8 weeks after irradiation. Support for loss of ovarian negative feedback as the cause of the increased $\mathrm{LH}$ and FSH concentrations comes from our observation of complete depletion of primordial follicles in the 30 and 50cGy groups at one week after irradiation. By 8 weeks after irradiation, nearly all the remaining primary, secondary and antral follicles would have already matured and ovulated or undergone atresia because the estimated time required for maturation of rodent follicles from the primordial to the ovulatory stage is 7-8 weeks (Hirshfield 1997). Increased production of $\mathrm{LH}$ and FSH due to pituitary tumors or other causes is very rare, and we feel that it is unlikely for the aforementioned reasons. However, in future studies, it would be useful to confirm that serum concentrations of estradiol and progesterone are low at 8 weeks after irradiation.

In conclusion, our results show that a 5 cGy dose of charged oxygen particle radiation, which is 8 -fold lower than the estimated cumulative radiation dose during a Mars mission of 40 cGy, decreases the primordial follicle pool by $71.5 \%$, while 30 cGy and 50cGy doses destroy essentially all primordial follicles. We compared the dose-response for primordial follicle depletion by oxygen ions in the present study to the dose-response for iron ions in our prior study (Mishra et al. 2016) and found that oxygen ions are more potent destroyers of primordial follicles than iron ions. Our results further show that follicle destruction by charged oxygen particles is preceded at $6 \mathrm{~h}$ after irradiation by dose-dependent increases in DNA double strand breaks, oxidative lipid damage and apoptosis in ovarian follicles. ALA supplementation was somewhat protective against induction of lipid peroxidation and apoptosis by 50 cGy charged oxygen particles, but this was insufficient to protect against follicle depletion. Our results raise concerns that exposure to space radiation may increase the risk for early ovarian senescence in female astronauts and that use of heavy ions in cancer radiotherapy may pose a greater risk for ovarian damage than conventional photon radiation. 


\section{Declaration of interest}

The authors declare that there is no conflict of interest that could be perceived as prejudicing the impartiality of the research reported.

\section{Funding}

Supported by National Aeronautics and Space Administration grant NNX14AC50G to $U$ L; National Institutes of Health grant P30CA062203, the University of California Irvine (UC Irvine) Chao Family Comprehensive Cancer Center; the Center for Occupational and Environmental Health, UC Irvine; the University of Virginia Center for Research in Reproduction Ligand Assay and Analysis Core, supported by the Eunice Kennedy Shriver NICHD/NIH (NCTRI) Grant P50-HD28934. B $M$ was partially supported by a First award fellowship from the National Space Biomedical Research Institute grant PF04302.

\section{Acknowledgements}

The authors thank Drs Peter Guida, Chiara La Tessa, Michael Sivertz and Adam Rusek, and staff at the NASA Space Radiation Laboratory for their expertise and support in carrying out the irradiations.

\section{References}

Barcellus-Hoff MH, Blakely EA, Burma S, Fornace AJJ, Gerson S, Hlatky L, Kirsch DG, Luderer U, Shay J, Wang Y et al. 2015 Concepts and challenges in cancer risk prediction for the space radiation environment. Life Sciences in Space Research 6 92-103. (doi:10.1016/j. Issr.2015.07.006)

Bourdarie S \& Xapsos M 2008 The near-earth space radiation environment. IEEE Transactions on Nuclear Sciences 55 1810-1832. (doi:10.1109/ TNS.2008.2001409)

Cadet J, Ravanat J-L, TavernaPorro M, Menoni H \& Angelov D 2012 Oxidatively generated complex DNA damage: tandem and clustered lesions. Cancer Letters 327 5-15. (doi:10.1016/j.canlet.2012.04.005)

Cooper RL, Goldman JM \& Vandenbergh JG 1993 Monitoring of the estrous cycle in the laboratory rodent by vaginal lavage. In Female Reproductive Toxicology, pp 45-55. Eds JJ Heindel \& RE Chapin. San Diego: Academic Press, Inc.

Cortés-Wanstreet MM, Giedzinski E, Limoli CL \& Luderer U 2009 Overexpression of glutamate cysteine ligase protects human COV434 granulosa tumor cells against oxidative and $\gamma$-radiation-induced cell death. Mutagenesis 24 211-224. (doi:10.1093/mutage/gen073)

Cucinotta FA \& Durante M 2006 Cancer risk from exposure to galactic cosmic rays: implications for space exploration by human beings. Lancet Oncology 7 431-435. (doi:10.1016/S1470-2045(06)70695-7)

Dayal D, Martin SM, Limoli CL \& Spitz DR 2008 Hydrogen peroxide mediates the radiation-induced mutator phenotype in mammalian cells. Biochemical Journal 413 185-191. (doi:10.1042/BJ20071643)

Dubey RK, Imthurn B, Barton M \& Jackson EK 2005 Vascular consequences of menopause and hormone therapy: importance of timing of treatment and type of estrogen. Cardiovascular Research 66 295-306. (doi:10.1016/j.cardiores.2004.12.012)

Hirshfield AN 1997 Overview of ovarian follicular development: considerations for the toxicologist. Environmental and Molecular Mutagenesis 29 10-15. (doi:10.1002/(SICI)10982280(1997)29:1<10::AID-EM2>3.0.CO;2-H)

ICRP 2003 Relative biological effectiveness (RBE), quality factor (Q), and radiation weighting factor $(\mathrm{WR})$. ICRP Publication 92. Annals of the ICRP 33 1-121. (doi:10.1016/s0146-6453(03)00024-1)
Kerr JB, Hutt KJ, Michalak EM, Cook M, Vandenberg CJ, Liew SH, Bouillet P, Mills A, Scott CL, Findlay JK et al. 2012 DNA damage-induced primordial follicle oocyte apoptosis and loss of fertility require TAp63mediated induction of Puma and Noxa. Molecular Cell 48 343-352. (doi:10.1016/j.molcel.2012.08.017)

Kim JK \& Lee CJ 2000 Effect of exogenous melatonin on the ovarian follicles in $\gamma$-irradiated mouse. Mutation Research 449 33-39. (doi:10.1016/ S0027-5107(00)00027-0)

La Tessa C, Sivertz M, Chiang I-H, Lowenstein D \& Rusek A 2016 Overview of the NASA space radiation laboratory. Life Sciences in Space Research 11 18-23. (doi:10.1016/j.Ifs.2016.02.037)

Lee CJ \& Yoon Y-D $2005 \gamma$-Radiation-induced follicular degeneration in the prepubertal mouse ovary. Mutation Research 578 247-255. (doi:10.1016/j.mrfmmm.2005.05.019)

Lee CJ, Park HH, Do BR, Yoon YD \& Kim JK 2000 Natural and radiationinduced degeneration of the primordial and primary follicles in the mouse ovary. Animal Reproduction Science 59 109-117. (doi:10.1016/ S0378-4320(00)00072-5)

Lim J, Lawson GW, Nakamura BN, Ortiz L, Hur JA, Kavanagh TJ \& Luderer U 2013 Glutathione-deficient mice have increased sensitivity to transplacental benzo[a]pyrene-induced premature ovarian failure and ovarian tumorigenesis. Cancer Research 73 908-917. (doi:10.1158/0008-5472.CAN-12-3636)

Lo Presti A, Ruvulo G, Gancitano RA \& Cittadini E 2004 Ovarian function following radiation and chemotherapy for cancer. European Journal of Obstetrics, Gynecology, and Reproductive Biology 113 S33-S40. (doi:10.1016/j.ejogrb.2003.11.008)

Lopez SG \& Luderer U 2004 Effects of cyclophosphamide and buthionine sulfoximine on ovarian glutathione and apoptosis. Free Radical Biology and Medicine 36 1366-1377. (doi:10.1016/j. freeradbiomed.2004.02.067)

Mathur S, Nandchahal K \& Bhartiya HC 1991 Radioprotection by MPG of mice ovaries exposed to sublethal gamma radiation doses at different postnatal ages. Acta Oncologica 30 981-983. (doi:10.3109/02841869109088253)

Meirow D \& Nugent D 2001 The effects of radiotherapy and chemotherapy on female reproduction. Human Reproduction Update 7 535-543. (doi:10.1093/humupd/7.6.535)

Mishra B, Ortiz L \& Luderer U 2016 Charged iron particles, typical of space radiation, destroy ovarian follicles. Human Reproduction 31 1816-1826. (doi:10.1093/humrep/dew126)

Molina JR, Barton DL \& Loprinzi CL 2005 Chemotherapy-induced ovarian failure. Manifestations and management. Drug Safety 28 401-416. (doi:10.2165/00002018-200528050-00004)

Pesty A, Doussau M, J-Lahaye B \& Lefèvre B 2009 Whole-body or isolated ovary 60Co irradiation: effects on in vivo and in vitro folliculogenesis and oocyte maturation. Reproductive Toxicology 29 93-98. (doi:10.1016/j. reprotox.2009.10.007)

Petersen Shay K, Moreau RF, Smith EJ \& Hagen TM 2008 Is $\alpha$-lipoic acid a scavenger of reactive oxygen species in vivo? Evidence for its initiation of stress signaling pathways that promote endogenous antioxidant capacity. IUBMB Life 60 362-367. (doi:10.1002/iub.40)

Petersen Shay K, Michels AJ, Li W, Kong A-NT \& Hagen TM 2012 Capindependent $\mathrm{Nrf} 2$ translation is part of a lipoic acid-stimulated detoxification stress response. Biochimica et Biophysica Acta 1823 1102-1109. (doi:10.1016/j.bbamcr.2012.04.002)

Rochette L, Ghibu S, Richard C, Zeller M, Cottin Y \& Vergely C 2013 Direct and indirect antioxidant properties of $\alpha$-lipoic acid and therapeutic potential. Molecular Nutrition and Food Research 57 114-125. (doi:10.1002/mnfr.201200608)

Ronca AE, Baker ES, Bavendam TG, Beck KD, Miller VM, Tash JS \& Jenkins M 2014 Effects of sex and gender on adaptations to space: reproductive health. Journal of Womens Health 23 967-974. (doi:10.1089/ jwh.2014.4915)

Shuster LT, Gostout BS, Grossardt BR \& Rocca WA 2008 Prophylactic oophorectomy in premenopausal women and long-term health. Menopause International 14 111-116. (doi:10.1258/mi.2008.008016)

Silva I, Mor G \& Naftolin F 2001 Estrogen and the aging brain. Maturitas 38 95-100. (doi:10.1016/S0378-5122(00)00195-X)

Slaba TC, Blattnig SR, Norbury JW, Rusek A, La Tessa C \& Walker SA 2015 GCR Simulator Reference Field and a Spectral Approach for Laboratory Simulation, NASA Technical Publication. Document number NASA/TP2015-218698. Langley, VA: NASA. 
Spitz DR, Azzam EI, Li JJ \& Gius D 2004 Metabolic oxidation/reduction reactions and cellular response to ionizing radiation: a unifying concept in stress response biology. Cancer and Metastasis Reviews 23 311-322. (doi:10.1023/B:CANC.0000031769.14728.bc)

Sridharan DM, Chappell LJ, Whalen MK, Cucinotta FA \& Pluth JM 2015 Defining the biological effectiveness of components of high LET track structure. Radiation Research 184 105-119. (doi:10.1667/ RR13684.1)

Suh JH, Shenvi SV, Dixon BM, Liu H, Jaiswal AK, Liu R-M \& Hagen TM 2004 Decline in transcriptional activity of Nrf2 causes age-related loss of glutathione synthesis, which is reversible with lipoic acid. PNAS 101 3381-3386. (doi:10.1073/pnas.0400282101)

Suit H, De LaneyT, Goldberg S, Paganetti H, Clasie B, Gerweck L, Niemierko A, Hall E, Flanz J, Hallman J et al. 2010 Proton vs carbon ion beams in the definitive radiation treatment of cancer patients. Radiotherapy and Oncology 95 3-22. (doi:10.1016/j.radonc.2010.01.015)

Svejme O, Ahlborg HG, Nilsson J-Å \& Karlsson MK 2012 Early menopause and risk of osteoporosis, fracture and mortality: a 34-year prospective observational study in 390 women. BJOG 119 810-816. (doi:10.1111/ j.1471-0528.2012.03324.x)
Tokuyama Y, Furusawa Y, Ide H, Yasui A \& Terato H 2015 Role of isolated and clustered DNA damage and the post-irradiating repair process in the effects of heavy ion beam irradiation. Journal of Radiation Research $\mathbf{5 6}$ 446-455. (doi:10.1093/jrr/rru122)

Wallace WHB, Shalet SM, Hendry JH, Morris-Jone PH \& Gattamaneni HR 1989 Ovarian failure following abdominal irradiation in childhood: the radiosensitivity of the human oocyte. British Journal of Radiology $\mathbf{6 2}$ 995-998. (doi:10.1259/0007-1285-62-743-995)

Wallace WHB, Thomson AB \& Kelsey TW 2003 The radiosensitivity of the human oocyte. Human Reproduction 18 117-121. (doi:10.1093/ humrep/deg016)

Received 19 February 2017

First decision 16 March 2017

Revised manuscript received 18 April 2017

Accepted 19 May 2017 